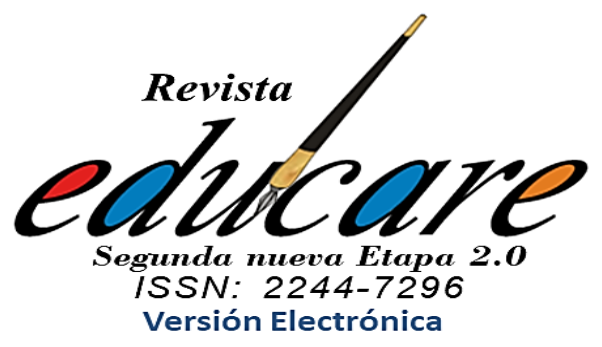

Volumen 25 No. 2 Mayo - Agosto 2021

$(60-82)$

\section{Isabel Teresa Suárez Pérez*}

ORCID: https://orcid.org/0000-0003-2353-4657

Jisson Oswaldo Vega Intriago**

ORCID: https://orcid.org/0000-0001-5727-8837

Kasandra Vanessa Saldarriaga Villamil ****

ORCID: https://orcid.org/0000-0002-8531-8346

Anicia Katherine Tarazona Meza****

ORCID: https://orcid.org/0000-0002-5126-3439

\section{Universidad Pedagógica Experimental Libertador \\ (Venezuela) \\ Universidad Técnica de Manabí (Ecuador)}

PhD en Educación. Docente Investigadora Jubilada de la Universidad Pedagógica Experimental Libertador, Instituto Pedagógico de Barquisimeto. Departamento de Educación Técnica. Miembro del Núcleo de Investigación Docencia, Innovación y Tecnología. E-mailisabels169@hotmail.com

* Doctor en Ciencias Psicológicas. Docente-Investigador. Universidad Técnica de Manabí, Departamento de Ciencias Sociales y del Comportamiento. Ecuador. Email: jisson.vega@utm.edu.ec

*** Licenciada en Ciencias de la especialidad Inglés. Magister en Administración de Empresas. Docente-Investigador de la Universidad Técnica de ManabíDepartamento de la Información. Ecuador. E-mail: kasandra.saldarriaga@utm.edu.ec

Licenciada en Ciencias de la Educación especialidad Psicología Educativa. Magister en Gerencia Educativa. Docente-Investigador de la Universidad Técnica de Manabí- Departamento de Pedagogía. Ecuador. E-mail: anicia.tarazona@utm.edu.ec

\section{PENSAMIENTO SISTÉMICO PARA EL DESARROLLO DE LA RESILIENCIA UNIVERSITARIA}

SYSTEMIC THINKING FOR THE DEVELOPMENT OF UNIVERSITY RESILIENCE
Recibido:

28-03-2021

Aceptado:

19-06-2021 


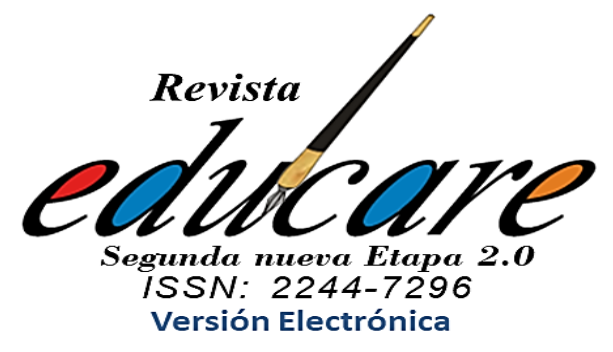

\title{
PENSAMIENTO SISTÉMICO PARA EL DESARROLLO DE LA RESILIENCIA UNIVERSITARIA
}

\author{
SYSTEMIC THINKING FOR \\ THE DEVELOPMENT OF \\ UNIVERSITY RESILIENCE
}

\section{Resumen}

Las universidades tienen que enfrentar situaciones adversas como la falta de recursos, poca capacidad de respuestas ante problemas como la desigualdad en el acceso a la educación, la ausencia de relaciones sistemáticas y programadas con el entorno, crisis sociales, económicas y sanitarias, todas estas situaciones han generado un colapso de los servicios, así como el cierre total o parcial de universidades. El escenario planteado constituye la motivación del presente trabajo cuyo objetivo es reflexionar sobre el pensamiento sistémico para el desarrollo de la resiliencia universitaria con el propósito de coadyuvar a la solución de los problemas universitarios. Se desarrolló bajo la modalidad documental se profundizó sobre el pensamiento sistémico, modelos epistémicas en los cuales está inmersa la Teoría General de Sistemas, la universidad como sistema y resiliencia estas teorías generaron unas recomendaciones para el desarrollo de la resiliencia en las universidades.

Palabras clave: Pensamiento sistémico; modelos epistémicos; universidad; resiliencia universitaria

\section{Abstract}

Universities have to face adverse situations such as lack of resources, little capacity to respond to problems such as inequality in access to education, the absence of systematic and programmed relationships with the environment, social, economic and health crises, all these situations They have generated a collapse of services, as well as the total or partial closure of universities. The proposed scenario constitutes the motivation of this work whose objective is to reflect on systemic thinking for the development of university resilience in order to contribute to the solution of university problems. It was developed under the documentary modality, it was deepened on systemic thinking, epistemic models in which the General Systems Theory is immersed, the university as a system and resilience, these theories generated recommendations for the development of resilience in universities.

Keywords: Systems thinking; epistemic models; university; university resilience 


\section{Introducción}

La educación universitaria es de suma importancia para el mejoramiento de la calidad de vida de un país, es por ello que la mejor inversión que pueden hacer las naciones es en la formación de profesionales capaces de hacer grandes transformaciones para la construcción de un mundo mejor. Al respecto, Tauber (2020) señala que:

Las universidades constituyen un elemento insustituible para el avance social, la generación colectiva de riqueza, el fortalecimiento de las identidades culturales, la cohesión social, la lucha contra la pobreza y el hambre, la prevención del cambio climático y la crisis energética, así como para la promoción de una cultura de paz, en la convicción que la búsqueda de la igualdad y la inclusión, a partir de una sociedad con igualdad de oportunidades de acceso al conocimiento. (p. 76).

El avance social se logra con universidades eficientes y equitativas, en las cuales su calidad esté directamente relacionada con la excelente formación de sus profesionales, en otras palabras cuando el sistema alcance mayoritariamente los objetivos que se había propuesto y cuando se beneficien equitativamente todos los individuos y grupos sociales, no olvidando que el principal objetivo de la educación superior es educar ciudadanos altamente calificados, con un gran espíritu de solidaridad y justicia social, (Mora, 2018).

Sin embargo, la eficiencia en las universidades se ve afectada por los grandes desafíos que les toca enfrentar, entre los cuales están: recursos financieros no adaptadas a las exigencias del mundo moderno, la poca capacidad de respuestas ante problemas como la desigualdad en el acceso a la educación, la ausencia de relaciones sistemáticas y programadas con otras instituciones de educación superior, con otros niveles educativos, con el estado y con sector empresarial, las crisis sociales, económicas y sanitarias, todas estas situaciones adversas han generado un colapso de los servicios, así como el cierre total o parcial de universidades. Es urgente que las universidades busquen mecanismos para contribuir significativamente a la construcción, fortalecimiento y consolidación de una sociedad más humana y a la formación de profesionales altamente calificados que se caractericen por la capacidad de transferir sus conocimientos y utilizarlos para el bienestar social (Pino, 2013). 
Las acciones que emprendan las universidades para solventar las situaciones planteadas las conducirán al cumplimiento de su misión, por lo tanto, se necesita cambiar la forma individualista a un pensamiento sistémico que conlleve a la comprensión de la realidad y a construir y reconstruir conocimientos más holísticos basándonos en el todo y no en una concepción reduccionista.

El pensamiento sistémico se desarrolla al considerar las conductas y fines individuales, las maneras en que las partes se combinan o relacionan mutuamente, las formas en que el sistema se relaciona con su medio ambiente y la configuración en subsistemas de menor jerarquía, entendiendo en todo momento que las interrelaciones de las partes y los esfuerzos de todos dan resultados superiores a los rendimientos individuales, (Marco y Loguzzo, 2012).

Tener un pensamiento sistémico en las universidades permitirá a las personas involucradas aceptar los cambios y transformaciones como oportunidades de aprendizaje y crecimiento, conllevará a detectar más claramente los problemas, las causas y consecuencias, entendiendo que los miembros son parte de un todo, que existe una conexión entre ellos y la realidad observada. El pensamiento sistémico conlleva a la aplicación de una nueva forma de gestión caracterizada por la resiliencia como mecanismo para la aceptación, adaptación y superación de las situaciones adversas. Es este sentido es oportuno mencionar a Cruz et al. (2021) quienes explican que la resiliencia es:

La capacidad que tiene una organización para anticipar, prepararse, responder y adaptarse al cambio y a interrupciones repentinas para sobrevivir y prosperar. Va más allá de lo que llaman gestión de riesgos, vista ésta como el proceso de identificar, analizar y responder a situaciones inesperadas, para asumir un enfoque más holístico de la salud y el éxito organizacional. Una organización resiliente no es solo aquella que sobrevive a largo plazo (que también es un aspecto deseado), sino aquella que se fortalece pasando las adversidades y cambios que se presentan en las pruebas del tiempo y se mantiene, logrando fortaleza en cada nuevo reto ( $\mathrm{p}$. 393).

Es importante que las universidades desarrollen su capacidad de resiliencia con el fin de salir airosos de las situaciones adversas y estar preparados para futuras eventualidades que le impidan el cumplimiento de sus objetivos, que es la formación de un recurso humano altamente calificado que transforme la sociedad en pro del bienestar individual y colectivo, este 
planteamiento constituye la motivación del presente trabajo cuyo objetivo es reflexionar sobre el pensamiento sistémico para el desarrollo de la resiliencia universitaria con el propósito de coadyuvar a la solución de los problemas universitarios.

\section{Desarrollo}

\section{Pensamiento sistémico}

El pensamiento sistémico es la tendencia de los individuos de percibir la realidad como un todo, esta forma de pensar se robustece con la Teoría General de Sistema de Ludwing Von Bertalanffy (1987), la cual se explicará más adelante luego de abordar los modelos epistémicos que la sustentan. Los modelos epistémicos son construcciones que muestran una serie de elementos y características para la comprensión de los fenómenos que emergen de la realidad, adoptando formas distintas atendiendo al conocimiento que representen, sus características y propiedades.

La epistemología a través de los modelos busca la representación y comprensión de las bases en las cuales se apoya la generación de los conocimientos; proviene de la voz griega epísteme, término usado por Sócrates para diferenciar el conocimiento científico entre el pensamiento y la técnica, se encarga del estudio de los métodos y procedimientos para estructurar y ordenar el conocimiento. Es la rama de la filosofía, llamada la doctrina del saber, cuyo propósito es estudiar el origen y la validez del conocimiento, coadyuvar en la resolución de problemas científicos, construir y reconstruir teorías, dilucidar y sistematizar los preceptos teóricos y prácticos a través de diversos modelos y técnicas, (Katayama y Pulido, 2017; Esteban, 2018).

La epistemología utiliza diversos modelos para determinar la veracidad de los conocimientos, estos modelos representan una aproximación a la realidad observada basándose en conceptos previamente establecidos y verificables que además le permiten la generación de nuevas teorías.

Los modelos epistémicos tienen sus principios y leyes para percibir la realidad y conocer el mundo que nos rodea, son representación abstracta o factual del contexto: es una concepción de 
un hecho o evento que da las pautas para examinar a profundidad el entorno o circunstancias significativas. Estas representaciones se fundamentan el pensamiento y se clasifican en originarios y derivados, los originarios están en la raíz de los conocimientos como son el naturalismo, idealismo, realismo y humanismo y los derivados son representaciones epistémicas que nacen de los originales, (Norman, Angarita y Díaz, 2020; Barrera, 2010).

El presente trabajo se fundamenta en el modelo epistémico originario del naturalismo y como modelos derivados el funcionalismo, estructuralismo y sistemicismo con la utilización de la Teoría General de Sistema de Ludwing Von Bertalanffy, seguidamente se profundizará en cada uno de ellos. El naturalismo es un modelo epistémico que establece que la fuente del conocimiento es la naturaleza como orientadora de la actuación de las personas a nivel individual y social, en este modelo se hace énfasis en el mundo físico, en el ambiente, en la condición humana, en la observación del holos (contexto, entorno y contorno) en la reflexión e interiorización para la obtención de los conocimientos, (Barrera, 2010).

El funcionalismo considera que el conocimiento se origina de las funciones de cada uno de los elementos de la sociedad, parte de la idea de que estos poseen una función significativa para mantener su estabilidad y equilibrio, explica como el colectivo humano se vincula con los fenómenos sociales formando un todo integrado, funcional, coherente y equilibrado con el fin de satisfacer sus necesidades, lidiar con los conflictos e irregularidades y regir el equilibrio social, otorgando a sus partes, (Raffino, 2020). En funcionalismo como modelos epistémicos interpreta la realidad se interpreta de los vínculos, procesos, funciones y maneras de actuación e interrelación de la sociedad, si cada elemento cumple con sus funciones la sociedad es funcional, en caso contrario, se considera disfuncional.

Rodríguez (2018), indica que el estructuralismo es describir el sentido de las cosas a través del revelamiento de la estructura que subyace; es un modelo que considera al individuo y a la sociedad como un sistema en el cual sus elementos están relacionados e interdependientes. Asimismo, Rodríguez (2018) y López (2008) nombra a Lévi-Strauss (1995) quien señala que la producción de conocimientos requiere de: a) la observación de la realidad; b) la construcción del modelo de realidad que muestra su estructura a través de la percepción y descripción y c) el análisis 
de la estructura con sus características y posibilidades de evolución, luego de estos pasos se produce el llamado modelo teórico, el cual debe ser construido de manera tal que su funcionamiento pueda dar cuenta de todos los hechos observados, involucre todos los elementos del problema y sea capaz de predecir nuevos hechos.

El estructuralismo postula que la realidad es una estructura constituida por elementos que se relacionan y conforman un todo complejo, centra el conocimiento en la visión de un todo interrelacionado. El estructuralismo y en funcionalismo están profundamente vinculados, el primero explica la estructura y el segundo como funciona, no prevalece el uno sin el otro, cuando se generan conocimientos a través de la interpretación de la realidad se considera su funcionalidad y a partir de allí se comprende la estructura como un todo.

El funcionalismo-estructural, cuya figura emblemática fue Parsons (1902-1979), es una corriente que enfatizó la idea de que las sociedades modernas deben ser entendidas como un sistema social cuya idea básica es que cada parte cumple una función específica e indispensable para el desempeño, equilibrio, estabilidad e integración de la estructura y de su preservación en el tiempo; también esta corriente permite describir y entender de qué manera las sociedades pueden enfrentar problemas, e incluso crisis y tener el poder de cambiar, (Salas 2020).

En lo que respecta a los sistemas sociales Talcott Parsons (1951) los define como:

Un sistema social (reducido a los términos más simples) consiste, pues, en una pluralidad de actores individuales que interactúan entre sí en una situación que tienen, al menos, un aspecto físico o de medio ambiente, actores motivados por una tendencia a obtener un óptimo de gratificación y cuyas relaciones con sus situaciones (incluyendo a los demás actores) están mediadas y definidas por un sistema de símbolos culturalmente estructurados y compartidos. (p. 7).

De igual manera el autor señalado indica que existen cuatro subsistemas que se corresponden con los imperativos funcionales: Subsistema social (integración); Subsistema Cultural (latencia); Subsistema de la personalidad (Capacidad para alcanzar metas) y Organismo Conductual (Adaptación). El estructuralismo y funcionalismo de Talcott Parsons originó el sistemicismo el cual tiene su origen histórico en la Teoría General de Sistemas de Ludwig Von Bertalanffy (1976), quien en su obra original la define como "la formulación de principios válidos 
para los sistemas en general, sea cual fuere la naturaleza de sus elementos componentes y las relaciones o fuerzas reinantes entre ellos" (p. 37).

Ludwig Von Bertalanffy (1987) señala que la teoría general de sistema no se limita a entidades materiales en física, biología y otras ciencias naturales, sino que también, es aplicable a entidades inmateriales y heterogéneas en alto grado incluye hombres con buena voluntad, lo que implica que tienen valores que son más que biológicos y que trascienden la esfera del mundo físico, como son el lenguaje, las relaciones simbólicas con sus semejantes, status social, leyes, ciencia, arte, moral. Este autor pretendió la compilación de todas las ideas referidas a sistemas para agruparlas en una única disciplina este propósito no se ha logrado, pero no cabe ninguna duda que los postulados de este autor han sido de utilidad en diversas disciplinas.

La teoría desarrollada por Karl Ludwig von Bertalanffy tenía una perspectiva interdisciplinaria, la cual denominó Teoría General de Sistemas orientada más en unir las cosas que en separarlas, en esta teoría se percibe la realidad como uno, integrado por elementos que no se pueden concebir por separado sino a través de su interrelación; esta constituye una manera de ver el mundo; una forma de hablar de la realidad; una herramienta de observación y de solución de problemas no se queda en determinar las características de las partes, incluye su análisis y comprensión, (Castro y Guzmán 2021). En ese mismo orden de ideas, De la Peña y Velázquez (2018) acotan:

En las diversas ramas del saber (humanísticas, ciencias exactas, de carácter tecnológico, ciencias médicas, informáticas, robótica, cibernética, entre otras) pudiera afirmarse que la teoría general de los sistemas y el enfoque sistémico tiene valor explicativo y argumentativo para la comprensión del funcionamiento en la realidad de los fenómenos y procesos, en particular para aquellos estudiosos e interesados en la construcción del conocimiento sobre la base de la manifestación de los fenómenos y procesos de la realidad objetiva en la que investigan, donde los sistemas de diferentes tipos están presentes y se requiere de la modelación de los mismos, su estructuración y funcionalidad hacia lo interno y entre sistemas de otras naturalezas en el plano externo.

La Teoría General de Sistemas constituye la base del pensamiento sistémico que es descrito por Lorenzon (2020) como: 
El pensamiento sistémico es integrador, tanto en el análisis de las situaciones como en las conclusiones que nacen a partir de allí, proponiendo soluciones en las cuales se tienen que considerar diversos elementos y relaciones que conforman la estructura de lo que se define como "sistema", así como también de todo aquello que conforma el entorno del sistema definido como ambiente. Podemos decir que el pensamiento sistémico se basa en cuatro conceptos fundamentales: • La interacción entre los elementos de un sistema • La globalidad, un sistema está compuesto de elementos donde el conjunto es superior a la suma de las partes (apareciendo las nociones de emergencia y de jerarquía). • La organización considerada como el concepto central del pensamiento sistémico. (p. 66).

En lo referente a los elementos que forman parte de la Teoría General de Sistema se expondrán de manera resumida los reseñados en la obra Sistemas y Organizaciones de Lorenzon (2020), este abordaje de conceptos permitirá ampliar los conocimientos para hacer uso del pensamiento sistémico en el desarrollo de la resiliencia universitaria.

- Un sistema abierto es un grupo de partes y objetos que interactúan de forma dinámica formando un todo relacionado con su medio ambiente, es aquel cuyas entradas se originan en el ambiente y cuyas salidas se vuelcan a él.

- Estructura son las interacciones e interrelaciones más o menos estables entre las partes o componentes de un sistema, que pueden ser verificadas e identificadas en un momento dado.

- Elemento son las partes o componentes que constituyen un sistema dentro de sus fronteras, estos pueden ser conceptos, sujetos, objetos o procesos.

- Frontera o límite es la línea que separa al sistema de su ambiente, que define lo que le pertenece y lo que queda fuera de él.

- Metasistema, contexto e intorno. Metasistema es todo aquello que se encuentra fuera de la frontera del sistema se denomina también entorno o medio ambiente. Contexto es la parte del metasistema que influye en el sistema.

- Intorno: Contexto interno del sistema. Está compuesto por todos los componentes internos y sus interrelaciones.

- La organización y control del sistema es el conjunto de reglas que condicionan el funcionamiento de los componentes de un sistema para el logro de los objetivos. 
- Subsistema son un conjunto de elementos y relaciones que responden a estructuras y funciones especializadas dentro de un sistema mayor.

- Entradas (Input): Todo sistema abierto requiere de recursos de su ambiente. Se denomina input a la importación de los recursos necesarios (Materia, energía e información) requeridos para realizar las actividades.

- Salidas (Output): Las salidas de los sistemas son los resultados que se obtienen al procesar las entradas. Resultan del funcionamiento del sistema y del propósito para la cual fue creado.

- El proceso total del sistema es el resultado de todas las actividades que convierten las entradas en salidas.

- Retroalimentación "Feed-back": Son los procesos mediante el cual se recoge información sobre los efectos del sistema para la toma de decisiones sobre el funcionamiento del mismo.

- Homeostasis: Son las compensaciones internas del sistema ante las variaciones del ambiente bloqueando o complementando los cambios con el objetivo de conservar la estructura sistémica.

- La entropía es la propiedad de degeneración de un sistema o la tendencia a la deformación funcional por el transcurso del tiempo y por el desgaste de la reiteración de los procesos.

- Modelo: Es la representación de la realidad, con su objetivo, funcionalidad y estructura. Toda realidad tiene la posibilidad de ser representado en más de un modelo. Un modelo es una abstracción de la realidad que captura la esencia funcional del sistema, con el detalle suficiente como para que pueda utilizarse en la investigación y la experimentación.

\section{La Universidad Como Sistema}

El sistema universitario como toda organización está compuesto de subsistemas que le permiten su funcionamiento interno y su relación con el medio ambiente, en este sentido es oportuno acotar los aportes realizados por Kast y Rosenzwwig (1987) en su obra Administración 
en las organizaciones un enfoque de sistema cuyas contribuciones teóricas son de especial connotación en el presente trabajo, estos autores señalan que toda organización es:

Un subsistema inserto en su medio y orientado hacia ciertas metas (gente con propósito), incluyendo un subsistema técnico (gente que utiliza conocimientos, técnicas, equipos e instalaciones); un sistema estructura (gente que trabaja junta en actividades integradas) un sistema psicosocial (gente que se interrelaciona socialmente) y que es coordinada por un subsistema administrativo (gente que planifica y controla el esfuerzo global). (p. 22)

La definición anterior indica que toda organización es un subsistema inmerso en un sistema mayor, engloba los diversos subsistemas interrelacionados que permiten el logro de los objetivos y el subsistema administrativo es el encargado de coordinar el esfuerzo de todos para de esta manera lograr que la organización tenga éxito. En ese orden de ideas Marco y Loguzzo (2012) expresan que:

Referirse a la organización como un sistema implica que se encuentra compuesta por un conjunto de elementos interrelacionados e interdependientes, que conforman una totalidad organizada con propiedades y características emergentes que no se encuentran en ninguno de sus elementos considerados aisladamente. A su vez, la definimos como un sistema abierto, ya que está en constante interacción con el contexto que la rodea. Es un sistema social vivo y dinámico que se recrean permanentemente, se reestructuran y se reinventan a medida que la dinámica organizacional va incorporando fines, alcanzándolos, incorporando tecnología, estableciendo nuevos objetivos o modificando sus estrategias. (p. 30).

En lo que respecta a la universidad como sistema social abierto esta tiene una serie de características que la define como tal, son un sistema interactivo complejos en su estructura y funciones, establecen relaciones de equilibrio internas y externas, está compuesto de subsistemas que contribuyen al funcionamiento y sometidos a constantes cambios y dichos cambios $\mathrm{y}$ transformaciones producen reestructuraciones en el sistema.

Además tiene las características expuesta por Lorenzon (2020) que les permite su funcionamiento y el cumplimiento de sus objetivos: permeabilidad (interacción con el medio ambiente); adaptabilidad (propiedad de un sistema de aprender y modificar un proceso, un estado o una característica de acuerdo a los cambios que sufre su contexto); viabilidad (medida de la 
capacidad de sobrevivencia y adaptación de un sistema a los cambios); armonía (propiedad que mide el nivel de afinidad de los sistemas con su ambiente); integralidad (nivel de coherencia interna en el cual un cambio producido en cualquiera de sus elementos producen cambios en los demás y hasta en el propio sistema); equifinalidad (se refiere al hecho que un sistema a partir de distintas condiciones iniciales y por distintos caminos llega a un mismo estado final) y organización (propiedad que tienen algunos sistemas de poder generar orden a partir del caos y es una parte esencial de cualquier sistema físico real.

\section{Desarrollo de la Resiliencia Universitaria}

Las universidades como sistemas sociales abiertos se ven afectados en lo interno y externo originando situaciones adversas que en ocasiones limitan el cumplimiento de sus objetivos y metas, es por ello que deben incorporarse a los modelos de gestión el reconocimiento de la incertidumbre y factores imprevisibles de carácter interno y externo que provocan alteraciones en las actividades y funcionamiento organizacional; este reconocimiento parte de la idea de que toda realidad puede ser modificada, en tanto la voluntad y la inteligencia colectiva decidan unificar esfuerzos en momentos de incertidumbre que requieren soluciones para solventar lo urgente y lo importante a través de estrategias de corto, mediano y largo plazo que funcionen en la normalidad y la emergencia, (Molea, 2020).

En los contextos de crisis universitaria los miembros de la comunidad comprenden la necesidad de desarrollar competencias en la toma de decisiones informada, resolución creativa de problemas y, sobre todo, adaptabilidad, capacidad de trabajo en contextos turbulentos y la resiliencia como habilidades a desarrollar en nuestros futuros profesionales y científicos. (Moriñigo y Corti 2020). El término resiliencia proviene de la física y se refiere a la capacidad de ciertos materiales de recuperarse a su forma original después de ser sometido a condiciones externas. En el campo de las ciencias sociales tiene una analogía similar al entenderse como la capacidad que tienen las personas de superar situaciones adversas y volver a su estado inicial o en mejores condiciones que las planteadas antes de la dificultad. 
$\mathrm{Al}$ respecto, Sánchez (2018) indica que:

El término resiliencia en las ciencias humanas se atribuye a la capacidad del hombre para atravesar situaciones adversas, y lograr salir no solamente a salvo, sino aún transformados positivamente por la experiencia. Bajo un enfoque sistémico es la capacidad de un sistema de mantener sus funciones y estructura haciendo frente a cambios internos y externos, y de adaptarse de la mejor manera cuando tenga que hacerlo. En general, los conceptos anteriores se desprenden dos perspectivas de especial interés para la organización como son: La supervivencia de la organización frente a una crisis; y lo que la organización hace y cómo lo hace a fin de prepararse y adaptarse a la realidad y entornos cambiantes. (p. 17)

La resiliencia organizacional es la habilidad para absorber y adaptarse en un entorno en constante cambio para cumplir sus objetivos y prosperar, para ello debe cumplir con los siguientes principios: comportamiento alineado con la visión, misión y valores, entendimiento del contexto, absorber, adaptarse y responder efectivamente al cambio, buen gobierno y gestión, diversidad de habilidades, liderazgo, conocimiento y experiencia, coordinación de todas las áreas y gestión del riesgo, (Organización Internacional de Normalización [ISO 22316], 2017)

La resiliencia es la capacidad de una organización para absorber y adaptase en un contexto variable con el propósito primordial de cumplir con sus metas y superar las situaciones problemáticas para poder sobrevivir y prosperar. Es un nuevo paradigma entre las organizaciones para afrontar un mundo futuro de incertidumbre, transformaciones y cambio, (Quiñonez y Prado, 2017). En ese mismo orden de ideas, Rogel y Urquizo (2019) plantean que:

La resiliencia es una habilidad que le sirve a las organizaciones para sobreponerse ante circunstancias adversas de cualquier índole, salir bien librada y quedar en mejores condiciones que antes de la crisis, es decir se convierte en una estrategia para el fortalecimiento de la cultura organizacional, y el desarrollo de capacidades gerenciales ante el riesgo. Es fundamental la capacidad que tiene el ser humano para enfrentar, sobreponerse y fortalecerse en las situaciones difíciles o de adversidades presentes en un momento determinado. Tener una actitud resiliente, implica hacerse de una visión distinta del mundo que rodea al individuo y la organización, la misma implica el aumento de las posibilidades de mantener relaciones de solidaridad para enfrentar los conflictos propios del ser humano. (p. 113)

En lo que respecta a la resiliencia universitaria bajo un pensamiento sistémico implica que estas instituciones de educación superior perciban al mundo en términos de totalidad, esta forma 
de pensar induce a comprender la realidad como un todo, en la cual están interrelacionados y todos son necesarios para la solución de las situaciones adversas y que el compromiso y actuación de cada miembro influye en el éxito o fracaso de la organización. En todo sistema y en lo que respecta al universitario existen los llamados imperativos funcionales del sistema expuestos por Parsons, (1951) y que se adaptan al concepto de resiliencia, como son: a) Adaptación ante situaciones adversas, (b) Capacidad para alcanzar metas fundamentales; (c) Integración que es la interrelación de sus componentes y (d) Latencia (mantenimiento de patrones); un sistema debe proporcionar, mantener y renovar la motivación de los individuos y las pautas culturales que lo integran.

Además, la resiliencia coadyuva a las universidades a cumplir con los fines de todo sistema como son la permeabilidad, adaptabilidad, viabilidad, armonía, integralidad, equifinalidad y la organización esto conllevará a estas instituciones de educación superior a cumplir con la misión para la cual fueron creadas, como es la formación de recursos humanos altamente calificados.

\section{Recomendaciones para el Desarrollo de la Resiliencia Universitaria}

A continuación, se mostrará una serie de recomendaciones para el desarrollo de la resiliencia universitaria tomando en consideración el pensamiento sistémico, sin pretender cerrar las posibles modificaciones producto de reflexiones posteriores, de igual manera estas recomendaciones se realizan cuando launiversidadpresente situaciones de cambios y transformaciones que impidan su normal desenvolvimiento, por lo tanto deben estar enmarcada en las políticas generales de la organización.

Las recomendaciones están formuladas para el subsistema administrativo en vista de que éste coordina los esfuerzos de los otros subsistemas, además se formularan atendiendo a la Teoría General de Sistemas, describiendo preliminarmente que es un sistema y sus elementos, los procesos administrativos de planificación, organización, dirección y control y evaluación y por último se abordará al liderazgo resiliente como el motor para cumplir con la misión de la universidad. Atendiendo a lo expuesto, Marco y Loguzzo (2012) expresan que: 
Un sistema se define como un conjunto de partes o elementos interrelacionados, que interactúan entre sí de acuerdo a determinada estructura. Es decir, los sistemas constituyen un todo organizado, en el cual la modificación de uno de sus elementos repercute produciendo cambios en el resto. El efecto total de un estímulo sobre el sistema total se verifica como un ajuste del sistema a las perturbaciones provocadas por ese estímulo. Los sistemas pueden clasificarse en cerrados y abiertos. Los sistemas cerrados son aquellos que no tienen vinculación con su medio, son herméticos y no reaccionan a los cambios en el entorno. Los sistemas abiertos, en cambio, están en constante interacción con su medio tomando recursos (información, materiales, energía) de él, transformando esos insumos y devolviendo un producto al entorno. (p. 29).

Señalan los autores de la cita anterior que los elementos de un sistema son: 1) Las entradas, recursos que requiere la organización para dar inicio a las operaciones del sistema; 2) Procesos de transformación el conjunto de actividades y operaciones que transforman las entradas en productos o servicios; 3) Las Salidas los resultados que el sistema arroja como producto o servicios luego de ejecutar los procesos; 4) La retroalimentación (feed back) la revisión del cumplimiento de las metas propuestas para establecer las correctivos de ser necesario, este es un mecanismo de monitoreo del accionar organizacional, que procura ajustar el desempeño de la organización con el propósito de una mayor efectividad y 5) El suprasistema o ambiente es la relación reciproca del sistema con el entorno; es el conjunto de fuerzas que rodean a la organización y afecta las operaciones internas de la misma.

Los procesos administrativos están relacionados con la planificación, organización, dirección y control y evaluación. La planificación determina los objetivos, los recursos que se requieran además de las actividades que se ejecutarán para logar alcanzar las metas planteadas: La organización en este proceso se fija la relación entre capital humano y económico con el que cuenta una organización para lograr alcanzar los objetivos y metas que se han proyectado en el respectivo plan. La dirección es la responsabilidad de los gerentes o líderes de guiar e instruir a las personas en el cumplimiento del trabajo asignado y el control es la evaluación y corrección de las actividades que realizan los subordinados para confirmar que lo que desarrolla o ejecuta está acorde al plan asignado, (Obregón y Ponce 2018). 
La persona que dirige la organización cobra especial importancia para el cumplimiento de todos los procesos es por ello que se necesitan de líderes resilientes para conducirla al éxito después de transitar por situaciones adversas. al respecto Mendoza (2020) señala que "un líder resiliente es visto de modo positivo por el grupo, pues manifiesta capacidad para interpretar sus anhelos, cualidad requerida para el ejercicio de la gerencia con un liderazgo comprensivo, la cual garantiza un trabajo en equipo efectivo, modalidad que se impone sobre el desempeño individual” (p. 9)

Según Sánchez (2020):

La resiliencia es ni más ni menos la capacidad de un sistema, un cuerpo, una materia, una persona, un equipo o una empresa, para absorber y soportar golpes, perturbaciones, traumas, adversidades, situaciones de alto riesgo y estrés, y recuperarse. La resiliencia y el juicio moral son dos factores fomentadores de conductas y actitudes de liderazgo personal y social, y contribuyen de sobremanera en ejercer un verdadero liderazgo, influyente, inspirador y coherente, uno que permita y contribuya con un desarrollo a nivel individual y en equipo, no solo por parte del líder sino también de los seguidores, (p. 83-84).

Los estilos de liderazgo están cambiando hacia una nueva forma de gestión en vista de las grandes transformaciones que ocurren a nivel mundial, se necesita de líderes resilientes que se adapten rápidamente a los cambios y coadyuven a las organizaciones a cumplir a misión para la cual fueron creadas. En la siguiente figura se visualiza todo el sistema para el desarrollo de la resiliencia universitaria y posteriormente se explicará cada uno de los elementos: 


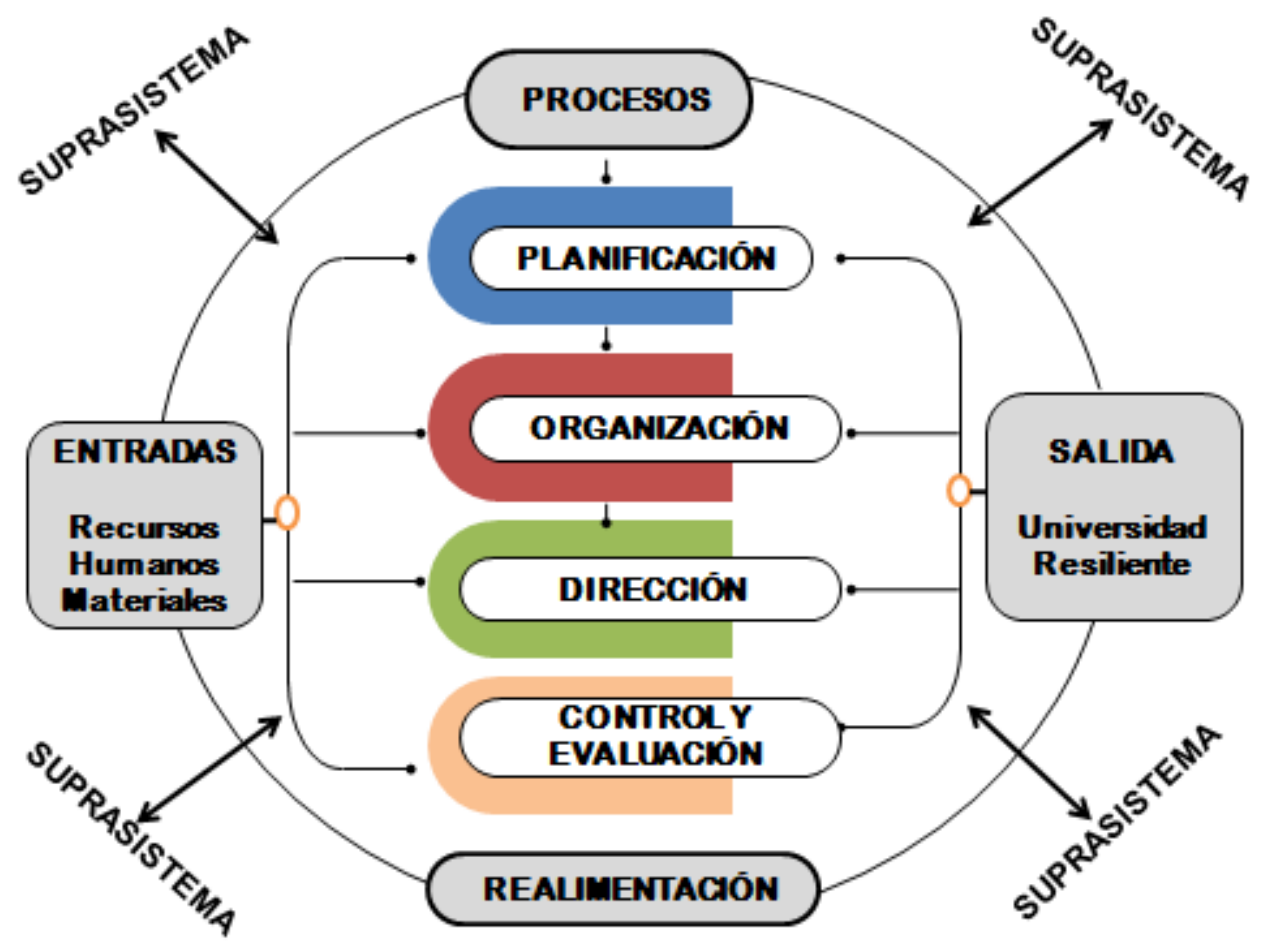

Figura 1 Modelo sistémico para el desarrollo de la resiliencia organizacional

\section{$\underline{\text { Entradas }}$}

Recursos Humanos con las siguientes competencias resilientes:

Recomendaciones

- Tener una mentalidad estructurada y funcional para precisar su visión, misión, valores y objetivos de la universidad

- Desarrollar habilidades creativas y de flexibilización ante los cambios

- Salir de su zona de confort, reconocer y se adaptarse a la situación de incertidumbre para activarse en el cumplimiento de los objetivos universitarios.

- Desarrollar su inteligencia emocional

- Ser flexibles en sus opiniones y respetar las opiniones de los demás

- Ser perseverantes y tolerantes ante las situaciones adversas. 
- Confiar en sus capacidades.

- Mantener buenas relaciones sociales con los miembros de la comunidad universitaria interna y externa.

- Aceptar las dificultades y promover soluciones

- Detectar los problemas y las situaciones riesgosas que afecten el funcionamiento de la universidad.

- Ser comprometidas con la universidad y concentrarse en la tarea asignada.

- Ser creativos e innovadores.

- Aprovechar las situaciones adversas para lograr oportunidades de crecimiento y aprendizaje.

Recursos Materiales:

- Materiales, infraestructura, tecnológicos

- Financieros

- Legales

\section{$\underline{\text { Procesos }}$}

Se ejecutarán los procesos de planificación, organización, dirección y control y evaluación.

\section{Planificación}

Recomendaciones

- Realizar una reunión previa para reflexionar, comenzando con

- las siguientes preguntas ¿Cómo está la universidad? y ¿Cómo quiero que esté? El reconocimiento de la situación de la organización el primer paso para la identificación del problema, esto implica ubicarse en el ahora, entender, aceptar y adaptarse (no significa resignación) a la realidad, además evaluar los recursos internos y externos disponibles para 
inventar e innovar en la toma de y por último visualizarse como organización exitosa y resiliente.

- La universidad tiene sus valores, misión y visión establecidos previamente, sin embargo, en momentos de transformaciones y cambios es necesario su fortalecimiento y convertirlos en patrones de comportamiento con el propósito de unir a los miembros de la organización para salir de la situación problemática.

- Realizar un diagnóstico de la situación para: conocer la magnitud del problema, identificar las fortalezas, debilidades, amenazas y oportunidades, determinar los recursos humanos y materiales disponibles. La información que arroje el diagnóstico debe ser sistematizada para luego diseñar el plan de acción.

\section{Organización}

Recomendaciones

- Conformar equipos de trabajo y aplicar un programa de capacitación en competencias resilientes y en el uso de las tecnologías.

- Crear un comité de emergencia para canalizar los problemas, proponer mejoras y soluciones.

- Crear una estructura organizativa en la cual se divida el trabajo y se asignen actividades y responsabilidades.

- Dotar de recursos humanos y materiales para la ejecución del plan de acción.

\section{Dirección (Liderazgo resiliente)}

Recomendaciones:

- Incentivar al personal para que tengan la disposición de realizar las actividades en pro del bien común.

- Realizar actividades para el fortalecimiento de relaciones sociales efectivas.

- Promover el compromiso y la obtención de estándares elevados de desempeño

- Impulsar equipos de trabajos que unan sus esfuerzos para solventar la situación problemática. 
- Promover la cultura de resiliencia universitaria.

- Establecer una comunicación efectiva entre todos los niveles

- Diseñar mecanismos de información para dar a conocer los logros alcanzados.

- Fomentar la creatividad y la innovación

- A través del ejemplo generar confianza en su equipo de trabajo utilizando criterios de igualdad y justicia.

- Reconocer el esfuerzo que hacen los miembros de la organización para salir de la situación problemática.

- Fomentar los valores universitarios y fortalecer la solidaridad y cooperación.

- Promover actividades para el manejo de situaciones riesgosas.

- Aceptar la diversidad de criterios y opiniones entre los miembros del equipo

- Utilizar los mecanismos de negociación para crear ambientes que propendan a la generación de nuevas ideas.

\section{Control y Evaluación:}

Recomendaciones:

- Elaborar planes de evaluación del desempeño del personal universitario

- Medir los logros

- Evaluar la ejecución de las actividades y su impacto en la resolución de los problemas.

- Formular indicadores de gestión.

- Establecer correctivos en caso de ser necesarios.

\section{$\underline{\text { Salida }}$}

Universidad resiliente con un pensamiento sistémico e integrador que supere las situaciones adversas y se transforme en una mejor organización para cumplir con la misión para la cual fue creada.

\section{$\underline{\text { Retroalimentación o "Feed-back" }}$}


Son los procesos mediante el cual se evalúa al sistema para tomar decisiones sobre su funcionamiento en caso de ser necesarios debe realimentarse el sistema con el fin de lograr los objetivos organizacionales.

\section{$\underline{\text { Suprasistema }}$}

El Suprasistema ambiental establece las interrelaciones entre la organización y su medio, el cual le suministra insumos y acepta sus productos.

Recomendaciones

- Identificar en el entorno oportunidades para el crecimiento de la universidad.

- Establecer alianzas de cooperación con otras universidades para el intercambio de recursos y experiencias resilientes.

- Propiciar contactos con el estado a fin de lograr acuerdos para el cumplimiento de las normas legales en tiempo de dificultades.

- Estudiar el entorno con fin de visualizar nuevas oportunidades.

- Cumplir con la responsabilidad social para solventar problemas en el entorno.

\section{Reflexiones Finales}

Considerando lo expuesto anteriormente se puede inferir que las universidades como toda organización es un sistema social abierto que está conformada por gente con propósito, capacitada, que trabaja de forma integrada y se relaciona socialmente, forma parte de un todo cuyas elementos están interrelacionadas mostrándose como una institución holística, integrada, con dependencia recíprocas entre sus partes y con constante relación con su entorno, como toda organización afronta cambios y transformaciones originadas por su funcionamiento interno o por impactos que vienen fuera de su frontera, en estos casos cobra especial importancia la resiliencia para que las universidades salgan airosas de las situaciones adversas y pueda cumplir su razón de ser que es la formación de un recurso humano altamente calificado. 
De igual manera, la resiliencia universitaria bajo el modelo sistémico implica tener una visión de totalidad, en la cual se perciba al mundo como un todo interrelacionado en el cual cada persona tiene su compromiso en la preservación de la organización, esto implica un transitar entre la realidad presente y la visión de la realidad que se aspira tomando en consideración las interrelaciones internas y las informaciones del suprasistema. Las organizaciones "resilientes" necesitan de personas "resilientes", capaces de tener una visión sistémica e integradora que perciba el todo, las partes y sus interrelaciones, que entienda que sus acciones afectan positiva o negativamente a toda la organización.

\section{Bibliografía}

Barrera, M. (2005). Modelo Epistémico en Educación y en Investigación. (3ra. Ed.). SIPAL. Fundación Servicios y Proyecciones para América Latina. Venezuela.

Castro, S., \& Guzmán de Castro, B. J. (2021). Políticas educativas basadas en el pensamiento sistémico y la gestión del conocimiento. Revista EDUCARE - UPEL-IPB - Segunda Nueva Etapa 2.0, 25(1), 35-61. https://doi.org/10.46498/reduipb.v25i1.1346

Cruz Montero, J. M., Avila Vasquez, M., Bringas Salvador, J. L., \& Tejada Estrada, G. C. (2021). Programación NeuroLingüística y Resiliencia Organizacional. Revista Venezolana De Gerencia, 26(93), 384-396. https://doi.org/10.52080/rvg93.26

De la Peña, G., y Velázquez, R. (2018) Algunas reflexiones sobre la teoría general de sistemas y el enfoque sistémico en las investigaciones científicas. Revista Cubana de Educación Superior, $\quad 37(2) . \quad$ http://scielo.sld.cu/scielo.php?script=sci_arttext\&pid=S0257$\underline{43142018000200003}$

Esteban Nieto, N. (2018). Utilidad de la Nueva Epistemología, según Mario Bunge. UNISDGInstitucional. https://alicia.concytec.gob.pe/vufind/Record/UNIS_3f1f7270f3bb802dbe4b26c50c0e464 1 
Kast, F. y Rosenzweig, J. (1987). Administración de las Organizaciones. (4ta. Ed.). Editorial Mc.

Graw Hill. https://es.scribd.com/doc/284354482/Kast-Rosenzweig-Administracion-enLas-Organizaciones-Capitulo-5

Katayama, R., y Pulido, V. (2017). Epistemología. Universidad Inca Garcilaso de la Vega. http://repositorio.uigv.edu.pe/bitstream/handle/20.500.11818/1159/Epistemolog\%c3\%ada .pdf? sequence $=1 \&$ is Allowed $=\mathrm{y}$

López, M. (2008). Construcción del conocimiento. https://www.esap.edu.co/portal/wpcontent/uploads/2017/10/4-Construccion-del-Conocimiento.pdf

Lorenzon, E. (2020). Sistemas y Organizaciones. Teoría General de Sistemas. Facultad de Informática. Universidad Nacional la Plata. http://sedici.unlp.edu.ar/handle/10915/99629

Ludwing Von Bertalanffy (1976). Teoría General de los Sistemas. Fondo de Cultura Económica. Ediciones F.C.E.

Marco, F. y Loguzzo, H. (2012). Gestión y administración de las organizaciones. Florencio Varela, Argentina: Universidad Nacional Arturo Jauretche

Mendoza, N. (2020). Aproximación teórica al significado del liderazgo resiliente en la Universidad Pedagógica Experimental Libertador. Revista EDUCARE - UPEL-IPB - Segunda Nueva Etapa 2.0, 24(1), 6-27. https://doi.org/10.46498/reduipb.v24i1.1224

Molea, D. (2020). Elegimos seguir. En P. Falcón La universidad entre la crisis y la oportunidad. Editorial de la Universidad Nacional de Córdoba. https://www.unaj.edu.ar/wpcontent/uploads/2020/12/La-universidad-entre-la-crisis-y-la-oportunidad.pdf

Mora, J. (2018). Universidades: mitos, modas y tendencias. Revista Iberoamericana de educación $\begin{array}{llll}\text { superior. } & \text { Superior } & \text { 3-16. }\end{array}$ https://doi.org/10.22201/iisue.20072872e.2018.24.259

Moriñigo, V y Corti A. (2020) Acciones y desafíos de la UNSL durante el periodo de aislamiento social preventivo y obligatorio En P. Falcón La universidad entre la crisis y la oportunidad. Editorial de la Universidad Nacional de Córdoba. https://www.unaj.edu.ar/wpcontent/uploads/2020/12/La-universidad-entre-la-crisis-y-la-oportunidad.pdf 
Norman Gómez, R., Angarita Valencia, J., \& Díaz González, C. (2020). Modelos epistémicos, investigación y método. Revista Oratores, (13), 120-131. https://doi.org/10.37594/oratores.n13.416

Obregón, X. y Ponce, G. (2018). Propuesta de mejora de procesos administrativos aplicado a Cnel-Ep matriz Guayaquil. [Tesis - Ingenieria Comercial. Universidad de Guayaquil Facultad de Ciencias Administrativas]. http://repositorio.ug.edu.ec/handle/redug/27190 Organización Internacional de Normalización- ISO 22316. (2017). Resiliencia en las Organizaciones. $\quad$ https://www.globalsuitesolutions.com/es/iso-22316-resilienciaorganizacional/

Parsons, T. (1951) El Sistema Social. https://teoriasuno.files.wordpress.com/2013/08/el-sistemasocial-talcott-parsons.pdf

Pino, E. (2013). La dimensión social de la universidad del siglo XXI creación del programa de aprendiazaje-servicio en la Universidad Técnica de Ambato. [Tesis Doctoral. Universidad Complutense de Madrid, Facultad de Educación]. https://eprints.ucm.es/id/eprint/22393/1/T34660.pdf

Quiñonez, R, y Prado, M. (2017). Resiliencia Organizacional. Ideas para el debate en el contexto ecuatoriano. Dominio de las Ciencias, 3(Extra 3), 488-504. https://dominiodelasciencias.com/ojs/index.php/es/index

Raffino M, (2021) Funcionalismo https://concepto.de/funcionalismo/

Rodríguez, R. (2018). El estructuralismo como modelo epistémico que busca explicar la realidad social. Revista Venezolana de Análisis de Coyuntura, XXIV(2), 147-155. http://saber.ucv.ve/ojs/index.php/rev_ac/article/view/16867

Rogel Gutiérrez, E. M., \& Urquizo Villafuerte, J. I. (2019). Aproximación teórica a la resiliencia en las organizaciones financieras. Revista De Ciencias Sociales, 25(2), 112-119. https://doi.org/10.31876/rcs.v25i2.27340

Salas, D. (2020). Manual de epistemología para la investigación en negocios. Escuela de Negocias de la Universidad Católica $\quad$ del 


\section{https://cdncentrum.pucp.education/centrum/uploads/2020/10/30161105/libro-}

epistemologia.pdf

Sánchez, A. (2020). Liderazgo resiliente pertinente para una sociedad cambiante. Foro Educacional, (34), 77-103. https://doi.org/10.29344/07180772.34.2361

Sánchez, M. (2018). Resiliencia: alternativa de las organizaciones venezolanas. Revista Estudios Gerenciales y de las Organizaciones, 2(3). https://regyo.bc.uc.edu.ve/v2n3/art01.pdf

Suárez Pérez, I. T., Trueba Macías, B. A., Venegas Álvarez, G. S., \& Proaño Rodríguez, C. E. (2020). La pertinencia social universitaria ante el Covid 19. Revista EDUCARE - UPELIPB - Segunda Nueva Etapa 2.0, 24(2), 249-272. https://doi.org/10.46498/reduipb.v24i2.1329

Tauber, F. (2020) Avances y desafíos de la educación superior en tiempos de pandemia. En P. Falcón La universidad entre la crisis y la oportunidad. Editorial de la Universidad Nacional de Córdoba. https://www.unaj.edu.ar/wp-content/uploads/2020/12/La$\underline{\text { universidad-entre-la-crisis-y-la-oportunidad.pdf }}$ 